\title{
He-3, Pierre Morel and Me-Early Work on Anisotropic Superfluidity
}

\author{
Philip W. Anderson
}

Received: 15 December 2010 / Accepted: 15 March 2011 / Published online: 2 June 2011

(C) The Author(s) 2011. This article is published with open access at Springerlink.com

\begin{abstract}
The idea that there are alternative, anisotropic solutions of BCS equations, which might apply to He-3, surfaced independently in at least three places, one of which was Pierre Morel's thesis project (for the ENS, under me at Bell Labs) I was skeptical of quantitative estimates of transition temperatures and instead focused, with Pierre, on conceptual and experimental properties of such states.
\end{abstract}

Keywords He-3 $\cdot$ Superfluidity $\cdot$ Generalised BCS theory

In 1959 David Pines decided to move from his assistant professorship at Princeton to the University of Illinois, where his field of many-body physics was more appreciated. He, John Bardeen and, a little later Gordon Baym, would form a very strong nucleus in that field. At Princeton he left behind the second of his brilliant French graduate students, Pierre Morel (the first was Philippe Nozieres). Pierre was employed as the science attache by the French Consulate in New York and could not follow him to Illinois, so David bequeathed him to me-perhaps the greatest of the many great favors David has done for me in the course of a long association. Socially Pierre was a little rich for my blood (one of my most vivid memories of him and his very beautiful wife was their elegantly seeing us off on our cut rate Icelandair flight to Cambridge in Sept. 1961 with an enormous bouquet of flowers), but physicswise he was just what I needed, extremely able mathematically, yet not too enamored of his own ideas.

I had a problem all ready for him, the possibility of exotic BCS-like states having pairs with finite angular momentum and thus anisotropic gaps, which I had scribbled down in a notebook a few months previously (March 241959 to be exact). It is pretty clear that this is one of those ideas whose time had come-it broke out independently

P.W. Anderson ( $₫)$

Department of Physics, Princeton University, Princeton, USA

e-mail: pwa@princeton.edu 
Fig. 1 Philip W. Anderson (right) with Ryogo Kubo taken in the fifties

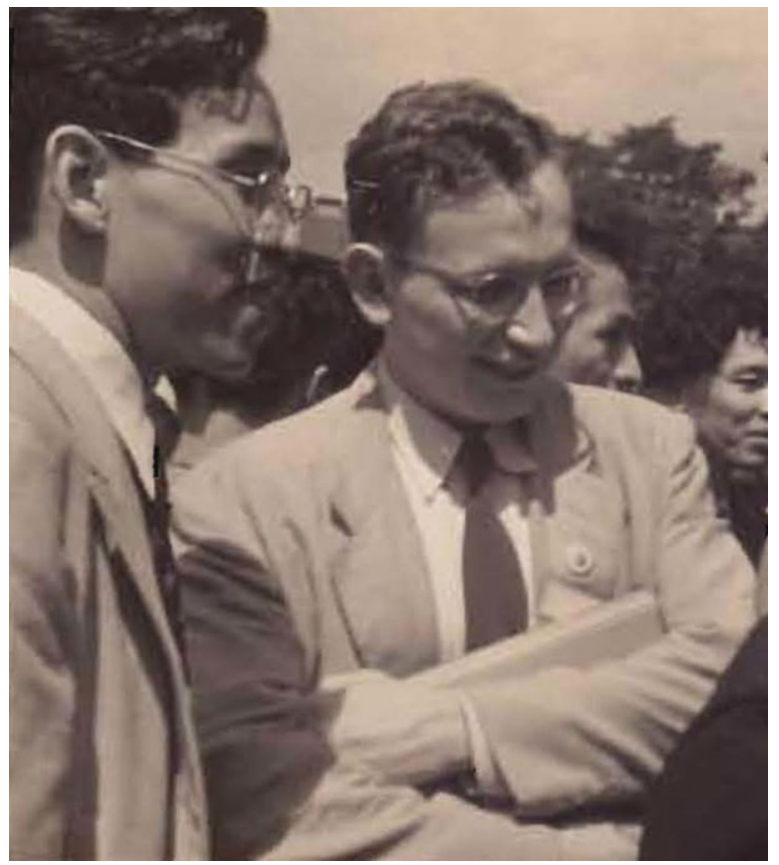

all over the place - and I don't think priority for it is very meaningful. In fact, the earliest publication was in Russian in JETP, 1959, by Lev Pitaevskii [1]; it played no role in the subsequent history in the West because none of us read it. Actually, even Pitaevskii does not claim credit for the basic idea, he in turn ascribes it to a casual remark by Landau. The Russians were from the first focusing on He-3, and Pitaevskii's paper spends most of its considerable length proving that some large enough $L$ will be attractive and therefore have a paired state in He-3. True enough.

In the West, one independent source of the idea was David Thouless, who writes about it as a small part of an enormous Annals of Physics review of BCS in 1960 [2]. He also mentions finite $L$ casually in his 1961 book, written in 1960. But David, like me at first, was unfamiliar with He-3 and never mentions that possibility. Although I referenced David in my early papers I-like, I am fairly sure, all the others involved-was totally independent: I had visited GE in Feb. '59 and heard a clumsy attempt at an anisotropic superconducting gap by a metallurgist named John Fisher, and my notebook contained how it should really be done. I learned about He-3 in the summer of ' 59 , from our summer visitor to Bell Labs Keith Brueckner, to whom I had explained my ideas, which he immediately realized would apply in that case.

I was a bit shocked to receive, less than two months later, a full-fledged paper from Keith and his graduate student Toshio Soda, in which he proposed not only an anisotropic state for He-3 but also a number of possibilities for nuclear matter. Since he had no idea such states were possible before talking to me, that seemed a bit much. I would normally have let it pass, but you must realize that this was my first ever graduate student and I was fiercely protective of him; Keith was quite nice about it and added our names as co-authors without cavil [3]. Keith, I suspect, had 
gotten word that Emery and Sessler were hot on our tail and was in a hurry. His submission date was Jan 1960; theirs a few weeks later. In the event, of course, we were all far behind Pitaevskii and it didn't matter. It is noteworthy that Emery and Sessler attribute the basic idea of finite $L$ to yet another source, Ben Mottelson.

Brueckner and Soda had one basic idea that was good and is central to the BCS theory in general. This goes back to the Bogoliubov concept that the BCS equation is a resummation of the ladder diagrams for scattering of quasiparticles near the Fermi surface. One can almost exactly sum up the high-energy scatterings and be left with a potential valid in a thin shell near the Fermi surface, which can be expanded in Legendre polynomials. $T_{c}$ is determined by a linear equation, which exactly separates out the different $L$ 's, and depends only on one parameter, equivalent to the scattering phase shift for the given $L$. They calculated this phase shift for different $L$ 's, using simply the quite well-known interatomic potential and making very crude allowances for the dense liquid surrounding the interacting atoms, and came out with a transition temperature in the neighborhood of .15 degrees $\mathrm{K}$.

I actually was dubious about claiming any particular transition temperature. What little was known at the time about He-3, and common sense, indicated that by any sensible measure it was more strongly-interacting by a lot than either typical metals or nuclear matter, and I felt sure that there were large renormalizations of all of its properties which many-body theory was not yet capable of coping with. Everything learned since then has confirmed this intuition. I think the last thing I heard, recently, was that it is still not possible from first principles to predict whether it will be triplet or singlet.

Nonetheless it was personally advantageous for Pierre that he was a co-author; a few years later, there was a brief period during which a low-temperature physicist in Russia claimed to see evidence for a transition in the $10 \mathrm{mdeg}$ range, and this coincided with a crucial promotion stage for Pierre within the French academic system-to Maitre de Conference, I believe. From that initial boost he went on to an extremely distinguished career in space physics to which He-3 was totally irrelevant.

What I thought was worthwhile in what we were doing was to establish some matters of principle: were such a state to occur, what would be its properties? From here on I will concentrate on our own papers, which were completely focused on these questions, not on supposedly realistic estimates of $T_{c}$. I took what I saw as an extremely bold step and chose to give my talk at the international congress in Utrecht (June, 1960) on this very speculative topic. At that conference there appears to have been a lot of discussion among David Thouless, Viktor Galitski and myself, and indeed in the official photograph (Fig. 2) two of us are to be seen sitting together. My paper, sent in July 1960, acknowledges Thouless and Galitskii; it is published in the Proceedings in Physica, vol. 26 [4]. Pierre and I also submitted a joint Phys. Rev. Letter, in July, and (in those faroff days) it appeared in August [5]. An erratum corrected a few (not all) egregious errors. Finally, Pierre and I published a full, and much more polished, paper in Phys. Rev. the next year, which was mostly on a possible $L=2$ state but mentioned in passing the possibility $L=1, S=1$.

Were these anisotropic states real superconductors? (In spite of the fact that they violated Landau's criterion for superfluidity, that excitations must have a minimum velocity or a gap. Many of them were gapless in that the gap had zeroes.) They were 


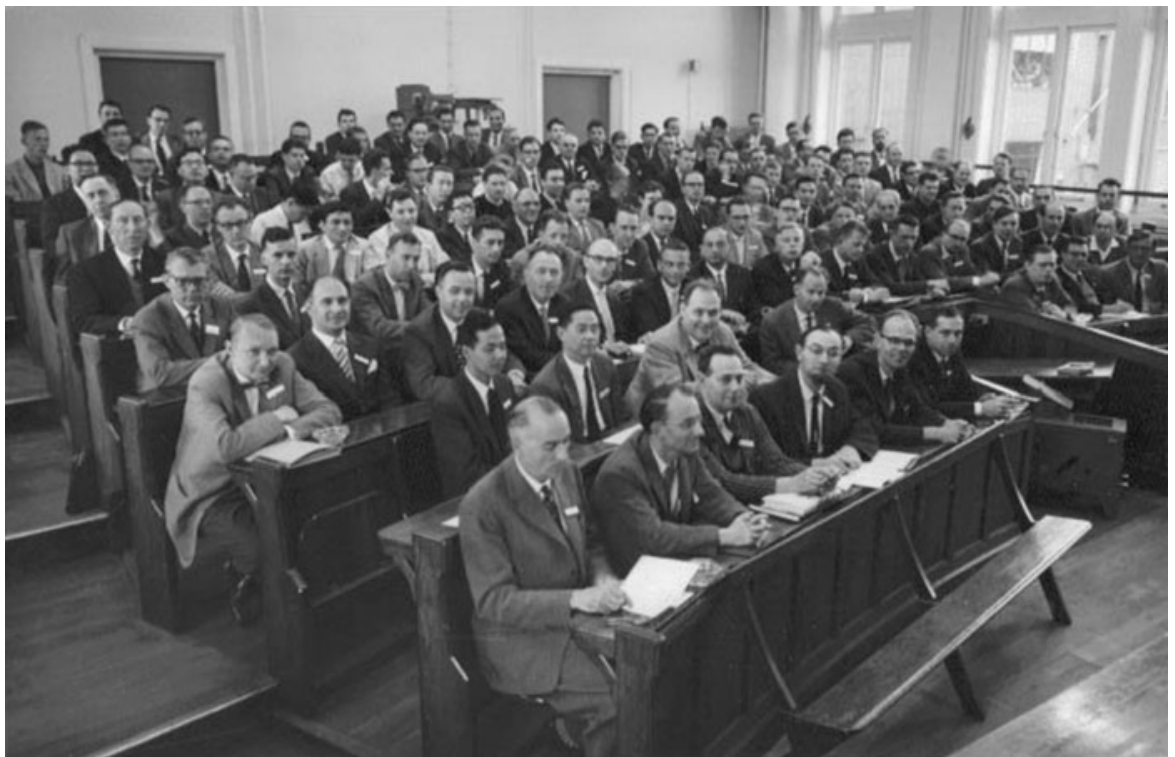

Fig. 2 Group photograph of the June 1960 Utrecht International Congress on statistical physics, at which I gave the first formal talk on anisotropic superfluidity. It was attended by several of the theorists who worked in this field then and later: Thouless, Sessler, Galitskii, Balian among them. Starting from the back, from left to right: Eighth row: ------- Cooper ------ Thouless, Brout, C. Bloch. Seventh row: Fukuda, Matsubara, Watson ------ ------ ------ ------ ---- Prigogine --- Lipkin. Sixth row: Uhlenbeck, Pais, Yang ----- Balian ----- Bohm ----- ----- ----. Fifth row: Domb, Meijer, de Domicis, P. Martin, Huang, Jacobsohn.... ---- ----- Schrieffer, Beliaev, Arai ----- Glassgold, Sessler. Fourth row: ----- Korringa -------- Zumino --Baur ------- P.W. Anderson, Galitski, Brown. Third row: Kramers, Careri, W.M. Fairbank, de Boer, de Groot, van Kampen, Siegert, Dabrovski, Hopfield, G. Chester. Second row: Lee, Lin, Green -----. Front row: Kronig, Suhl, Montroll, Klein, Van Hove, Pines. Further to right: Bogoliubov, Callen, Luttinger

indeed "super", and this was very enlightening as to the true nature of superfluidity. I still find Landau's criterion quoted in the textbooks, but it became meaningless in 1959. Also, they turned out to exhibit a number of different states for any given $L$ and $S$, all with the same $T_{c}$ because the equation for $T_{c}$ is linear, but with different free energies; how did they choose among them? What was the meaning of the apparent possibility of orbital ferromagnetism, and how would it manifest itself? In what sense would the system exhibit anisotropy? All of these questions really had to wait for final solution until we had the real stuff at hand, but we could begin to ask them.

\section{Superfluidity—Superconductivity}

Apparently Landau hung on rather desperately to his early contention that superfluidity was not related to quantum statistics, demonstrating that even great geniuses are capable of holding two incompatible opinions simultaneously-since he was also an author of Ginsburg-Landau with its complex order parameter. We used as our criterion for "superness" the Meissner effect, or equivalently the Andronikashvili effect for superfluids: that superflow is curlfree and derives from a coherent bosonic matter field. It is equivalent to the requirement of phase stiffness. 
Multiple States It is only very rarely, if at all, that BCS-type superconductivity exhibits a phase diagram of any complexity. We recognized from the first that a distinguishing feature of the anisotropic superconductors would be that whereas a BCS superconductor has the symmetry of the identity representation $\Gamma_{0}$ of the rotation group or of the point group of the crystal substrate, which is necessarily non-degenerate, the anisotropic superconductors will characteristically belong to degenerate representations, breaking the point-group symmetry in several possible ways. At the transition point, the equation determining $T_{c}$ is linear and thus all linear superpositions of these solutions have the same $T_{c}$ 's; but any non-linear effects distinguish among the substates. The solutions may, and usually will, also be complex, breaking time-reversal as well as the point-group symmetry.

Collective Modes I am pleased to have thought to mention these, apparently stimulated to an extent by Galitskii (see above); I had no idea what a big role they would play in the identification and understanding of the real thing, but at least I didn't leave them out.

In the early papers the only solutions we looked at were the individual $Y_{L m}$ 's for $m=0$ to $L$. We pointed out that the complete decoupling of the different $L$ 's was only valid right at $T_{c}$, but that in fact the effect of cross-coupling would be small if gaps were small compared to Fermi energies as we supposed was true. So one could calculate relative energies very well; the basic criterion is that the gap should cover the Fermi surface as completely as possible, which favors complex gaps because they have only point zeroes. (This is one of the most important mysteries of modern cuprate physics; why is the gap real? The answer says a lot about the physics [6].) At first, calculations seemed to favor the "orbital ferromagnets" with gap functions like $\sin \theta e^{\operatorname{im} \varphi}$, so we thought about total angular momentum, or more generally, what did a complex order parameter do? Our solution of that dilemma, which I still like, was to show that there is are easily calculable $\left\langle n(r) n\left(r^{\prime}\right)\right\rangle$ and $\left\langle n(r) J\left(r^{\prime}\right)\right\rangle$ correlations which latter can be shown not to contribute any bulk current but imply a small surface current, of order (gap)/(Fermi energy), which in turn implies a small angular momentum. Most of the rest of the discussion in those early papers was much too restricted as to the variety of possible states discussed, as we were to discover later. But I think we were the first to point out that the general phase diagram structure, with the overall $T_{c}$ the same or similar, but the possibility of a number of substates breaking the point-group symmetry in a number of different ways, was absolutely characteristic — and diagnostic — of this form of superconductivity.

In these papers we also pointed out a second diagnostic for these states: that in contrast to BCS superconductivity $T_{c}$ would be expected to be as sensitive to ordinary scattering as BCS is to magnetic scattering. This of course is no help in the case of He-3 since nothing dissolves in it (in one paper I made the solecism of stating that He-4 does, which is of course untrue). On the other hand, for some years after the discovery of heavy-electron superconductors which passed both of these criteria, as well as other criteria, also theoretically-based, the conventional wisdom seemed to be that nonetheless it was too radical to accept that they were of this exotic, unfamiliar, non-BCS class. Nowadays this unfortunate prejudice has evaporated, perhaps in the face of the cuprates, and it is possible to suggest as I have [7] that there may, in reality, be more non-BCS than BCS superconductors. 
Fig. 3 The pattern of surface currents implied by the $L=2$ superfluid state of Morel and Anderson, an observable manifestation of the complex order parameter

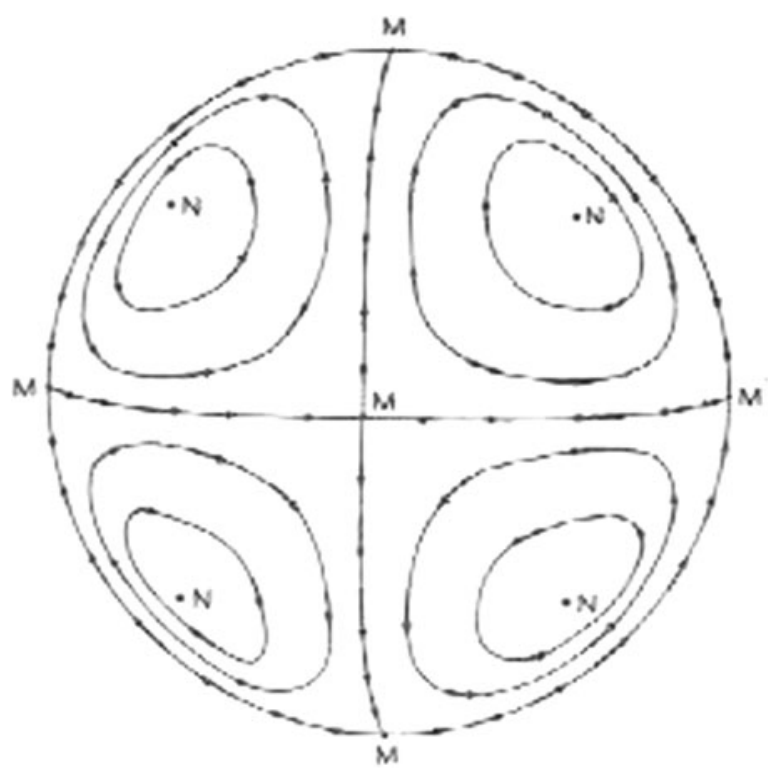

The third paper with Pierre Morel was a much more serious and thoughtful effort, suitable to be part of his thesis [8]. There is a thorough treatment of the pseudopotential idea and of the simplification of the potential. Here we mainly focused on possible d states, since at the time most people believed that $L=1 \mathrm{did}$ not avoid the hard-core repulsion sufficiently to be attractive. We discovered that the lowest energy state we could find is a complex superposition of $m=0$ and $m= \pm 2$, which breaks the rotational symmetry down to a representation of the group of a cube, and gives an energy gap magnitude of cubic symmetry. It does however break time-reversal symmetry and there would be circulating surface currents, though not such as to lead to a net angular momentum (Fig. 3). In passing, we carried along also the $L=1$ possibility, which was also complex, and did have a net angular momentum. We did not mention that the susceptibility for the $L=1$ AM state would be constant through $T_{c}$, which is obvious, but we did plot the d-wave susceptibility and showed that it was not. We also made a quantitative estimate of inelastic scattering effects on $T_{c}$, using the by now known real data on He-3, and concluded that the transition could not occur above $20 \mathrm{mdeg}$, and that the original estimate of $150 \mathrm{mdeg}$ had indeed been unrealistic.

This paper is, to my knowledge, the first to deal in some detail with the really complex questions of multiple symmetry-breaking; we took the problem seriously and found meat in it. Of course, we made one serious mistake, which Roger Balian and Dick Werthamer fixed [9]; and made one serious omission, the Fermi liquid corrections which Tony [10] supplied. And when the real He-3 appeared, it turned out that there was much more to do; but we made a brave start.

Open Access This article is distributed under the terms of the Creative Commons Attribution Noncommercial License which permits any noncommercial use, distribution, and reproduction in any medium, provided the original author(s) and source are credited. 


\section{References}

1. L.P. Pitaevskii, Zh. Eksp. Teor. Fiz. 37, 1794 (1959)

2. D.J. Thouless, Ann. Phys. 10, 553 (1960)

3. K.A. Brueckner, T. Soda, P.W. Anderson, P. Morel, Phys. Rev. 118, 1442 (1960)

4. P.W. Anderson, Physica 26, S137 (1960)

5. P.W. Anderson, P. Morel, Phys. Rev. Lett. 5, 136 (1960)

6. P.W. Anderson, Phys. Rev. Lett. 96, 017001 (2006)

7. P.W. Anderson, Ann. Henri Poincaré 4, 1 (2002)

8. P.W. Anderson, Pierre Morel, Phys. Rev. 123, 1911 (1961)

9. R. Balian, R. Werthamer, Phys. Rev. 131, 1553 (1963)

10. A.J. Leggett, Phys. Rev. 140, A1869 (1966) 